\title{
Improving outcomes for brain metastases in EGFR mutated NSCLC
}

\author{
Jennifer W. Carlisle, Suresh S. Ramalingam \\ Department of Hematology and Medical Oncology, Winship Cancer Institute, Emory University, Atlanta, GA, USA \\ Correspondence to: Suresh S. Ramalingam, MD. Roberto C. Goizueta Chair in Cancer Research, Department of Hematology and Medical Oncology, \\ Winship Cancer Institute, Emory University School of Medicine, 1365 Clifton Road NE, C-4014E, Atlanta, GA 30322, USA. \\ Email: ssramal@emory.edu. \\ Provenance: This is an invited article commissioned by the Section Editor Dr. Hengrui Liang, MD (Department of Thoracic Surgery, Guangzhou \\ Medical University, Guangzhou, China). \\ Comment on: Wu YL, Ahn MJ, Garassino MC, et al. CNS Efficacy of Osimertinib in Patients With T790M-Positive Advanced Non-Small-Cell Lung \\ Cancer: Data From a Randomized Phase III Trial (AURA3). J Clin Oncol 2018;36:2702-9.
}

Submitted Apr 25, 2019. Accepted for publication May 17, 2019.

doi: $10.21037 /$ tlcr.2019.05.08

View this article at: http://dx.doi.org/10.21037/tlcr.2019.05.08

Tyrosine kinase inhibitors (TKIs) of activating epidermal growth factor receptor (EGFR) mutations have revolutionized the care of patients with advanced non-small cell lung cancer (NSCLC) who harbor a targetable EGFR mutation. Compared to platinum doublet chemotherapy, median overall survival (OS) has improved from a historical 8-12 months with chemotherapy alone (1) to 26 months with first generation EGFR TKIs gefitinib and erlotinib (2). Patients treated with the first-generation EGFR TKIs have a 5 -year OS rate of nearly $15 \%$ in the real-world setting (3). In a recent analysis of the third generation EGFR TKI osimertinib in the $2^{\text {nd }}$ or $3^{\text {rd }}$ line setting at the time of acquired resistance with T790M in phase II trials, median OS was 26.8 months (4). While OS data in patients treated with the third generation EGFR TKI osimertinib in the frontline setting are currently maturing (5), long-term disease control is observed in a sizeable subset of patients. As patients are living longer with this disease, determining the optimal management of associated central nervous system (CNS) metastatic disease has become a pressing issue.

Wu et al. recently published a detailed report of the CNS efficacy of osimertinib in T790M positive NSCLC patients (6), as a pre-planned subgroup analysis of the AURA3 clinical trial (7). A total of 116 patients had CNS disease; among the 46 patients with measurable disease, the CNS objective response rate was $70 \%$ in the osimertinib group compared to $30 \%$ in the platinum pemetrexed group (6). Seven patients with suspected leptomeningeal disease were included in the osimertinib group, four of whom had either complete or partial radiographic response. Interestingly, 14 patients who received brain radiation within 6 months prior to initiation of osimertinib had a better CNS response rate (64\%) than those with more remote or no brain radiation (34\%) (6). This analysis demonstrates the efficacy of osimertinib in the treatment of patients with brain metastatic disease, including leptomeningeal disease.

A significant number of patients with EGFR mutated NSCLC develop brain metastases (BM) during the course of their disease. BM are identified at diagnosis in approximately $20-30 \%$ patients with EGFR mutated NSCLC $(8,9)$. In patients without BM at diagnosis, the actuarial rate of progressive disease in the brain was $6 \%$ at one year and $13 \%$ at two years based on a 100 patient cohort treated with gefitinib or erlotinib (8). In a similar cohort of 86 patients with advanced EGFR mutated NSCLC, the incidence of BM was $47 \%$ at 3 years, $49 \%$ at 4 years, and $53 \%$ at 5 years (10). A retrospective analysis comparing sequencing of therapy with the EGFR TKI erlotinib (98\% of patients) and either stereotactic radiosurgery (SRS) or whole brain radiotherapy (WBRT) suggested a favorable survival outcome with SRS followed by erlotinib (11). In patients treated with SRS first followed 
by erlotinib, the median OS was 46 months, whereas those treated with frontline erlotinib, median OS was only 25 months (11). These analyses were conducted in the era of first generation EGFR TKIs, which have a lower CNS penetration (12) and systemic efficacy then osimertinib (5).

There is limited preclinical and pharmacokinetic data detailing osimertinib penetration into the CNS. Ballard et al. demonstrated in rats, that radio-labeled osimertinib could be detected in the brain after a single dose and persist for 21 days with $4 \mathrm{mg} / \mathrm{kg}$ of osimertinib, while gefitinib only showed scant CNS uptake that was undetectable at $24 \mathrm{~h}$ with $5 \mathrm{mg} / \mathrm{kg}$ dosing (12). A positron emission tomography study also demonstrated markedly more CNS uptake in the brain of cynomolgus monkeys with osimertinib compared to gefitinib (12). While all of the currently available EGFR TKIs are substrates for efflux transporters (12), chemical properties that allow influx can overcome efflux (13). These principles help explain the lower mean CSF concentrations of gefitinib compared to erlotinib (14) and the penetration of osimertinib (13).

Additional information regarding the CNS efficacy of frontline osimertinib was recently published as a preplanned analysis of the FLAURA clinical trial (15). While baseline CNS imaging was not required, 61 patients treated with osimertinib and 67 patients treated with either gefitinib or erlotinib had known CNS disease at study entry (15). Patients treated with osimertinib had improved CNS progression free survival compared to patients treated with first generation EGFR TKIs (PFS not reached $v s$. 13.9 months respectively, HR 0.48 ; 95\% CI, 0.26-0.86; $\mathrm{P}=0.014)$. The objective response rate in the patients with at least one measurable lesion in 22 patients treated with osimertinib and 19 patients treated with first generation EGFR TKIs was $91 \%$ with osimertinib, compared to $68 \%$ in the control group (odds ratio 4.6; 95\% CI, 0.9-34.9; $\mathrm{P}=0.066$ ) (15). Encouraging results were also noted in the limited number of patients with radiographic evidence of leptomeningeal disease; 4 out of 5 patients treated with osimertinib demonstrated complete radiographic response, and the fifth had stable disease (15).

The impact of prior brain radiotherapy on the efficacy of osimertinib is not well established; of the 50 patients with baseline CNS disease treated as part of the AURA extension and AURA2 trial who were evaluable for response, 19 received radiation within the previous 6 months while 31 received no radiation or radiation over 6 months prior to osimertinib (16). In this analysis, patients with prior recent brain radiation had lower a CNS objective response rate of $32 \%$ compared to $68 \%$ with remote or no prior radiation (16). This is opposite of the trend seen in the AURA3 CNS analysis (6). In the FLAURA clinical trial, a total of 15 patients treated with osimertinib and 16 patients treated with first generation EGFR TKIs received brain radiation in the 6 months prior to study treatment, but only 6 and 9 respectively had a target lesion evaluable for response, limiting conclusions that can be drawn (15). While more data are awaited, current guidelines recommend consideration of osimertinib in patients with stable, asymptomatic CNS metastatic disease in lieu of upfront local therapy with SRS or WBRT $(17,18)$. This approach has the advantage of avoiding potential side effects of SRS and more importantly WBRT.

The presence of leptomeningeal disease is associated with a poor overall outcome and rapid decline in quality of life. In a recently reported expanded retrospective analysis of 22 asymptomatic patients with EGFR mutant NSCLC treated with osimertinib $80 \mathrm{mg}$ daily among the AURA clinical trial program, the leptomeningeal ORR was $55 \%$ (95\% CI, 32-76\%) and median OS was 18.8 months (95\% CI, 6.3-NC months) (19). A higher dose of osimertinib, $160 \mathrm{mg}$ daily, has also shown efficacy associated with levels of cerebral spinal fluid penetration in a study of 32 patients, and follow-up survival analysis is pending (20). Along with the reports of clinical benefit in the AURA3 and FLAURA clinical trials with leptomeningeal disease, there is now hope for this patient subset that has among the worst overall prognosis. The results of ongoing studies with specific focus on CNS disease will shed more light on the efficacy of osimertinib in patients with leptomeningeal disease as well as discrete brain metastatic disease (Table 1).

Care must be taken to avoid lumping patients with surgically resectable solitary metastatic disease or symptomatic disease into a frontline systemic TKI therapy plan, as such patients were generally not included in the clinical trials of osimertinib. In symptomatic patients with $\mathrm{BMs}$, the number, size and location of the metastasis guides the decision for SRS, WBRT, or surgical resection followed by SRS or WBRT. While the 2012 American Society for Radiation Oncology (ASTRO) guidelines recommend WBRT following resection (22), recent practice patterns have transitioned to post-operative SRS to the resection bed (23). For now, available data from the clinical development of osimertinib demonstrate CNS efficacy in patients with or without preceding brain radiation and oral TKI therapy is a well-tolerated option for patients with asymptomatic BMs and leptomeningeal disease. 
Table 1 Ongoing clinical trials of osimertinib for brain metastases and/or leptomeningeal disease (21)

\begin{tabular}{|c|c|c|c|c|}
\hline $\begin{array}{l}\text { ClinicalTrials.Gov } \\
\text { Identifier }\end{array}$ & Title & Phase & Number of patients & Expected completion date \\
\hline NCT03497767 & $\begin{array}{l}\text { A Randomised Phase II Trial of Osimertinib With or } \\
\text { Without Stereotactic Radiosurgery for EGFR Mutated } \\
\text { Non-Small Cell Lung Cancer (NSCLC) With Brain } \\
\text { Metastases }\end{array}$ & II & 80 & March 2022 \\
\hline NCT03769103 & $\begin{array}{l}\text { Open Label, Multicenter, Phase II Study of Patients } \\
\text { With Treatment Naïve Metastatic Epidermal Growth } \\
\text { Factor Receptor (EGFR) Mutation-Positive Non-Small } \\
\text { Cell Lung Cancer (NSCLC) With Brain Metastases } \\
\text { Randomized to Stereotactic Radiosurgery (SRS) and } \\
\text { Osimertinib or Osimertinib Alone }\end{array}$ & II & 76 & December 2022 \\
\hline NCT02736513 & $\begin{array}{l}\text { Pilot, Phase } 2 \text { Study Assessing Intracranial Activity of } \\
\text { AZD9291 (TAGRISSO) in Advanced EGFRm NSCLC } \\
\text { Patients With Asymptomatic Brain Metastases }\end{array}$ & II & 40 & May 2020 \\
\hline NCT03463525 & $\begin{array}{l}\text { An Open-label PET Study to Determine Brain } \\
\text { Exposure of Osimertinib After IV Microdose } \\
\text { Administration of }[11 \mathrm{C}] \text { Osimertinib and Therapeutic } \\
\text { Oral Doses of Osimertinib to Patients With EGFR } \\
\text { Mutated NSCLC With Brain Metastases }\end{array}$ & Not listed & 8 & September 2019 \\
\hline NCT02971501 & $\begin{array}{l}\text { A Phase II Trial of AZD9291 (Osimertinib) With or } \\
\text { Without Bevacizumab in Patients With EGFR Mutation } \\
\text { Positive NSCLC and Brain Metastases }\end{array}$ & II & 98 & July 2020 \\
\hline
\end{tabular}

The ability to achieve control of BM has played a major part in improving survival outcomes for patients with lung cancer; in other oncogene-addicted tumors such as with the anaplastic lymphoma kinase (ALK)-rearranged NSCLC, potent TKIs have resulted in improved control of BM $(24,25)$. This has been the result of development of potent TKIs that have the ability to achieve sufficient CNS drug levels. The advantages of using TKIs with potent CNS activity include improved long-term outcomes, avoidance of local therapy to the brain and overall preservation of quality of life for patients. As our knowledge of acquired resistance mechanisms to the new generation of TKIs improve, it is hoped that novel combination approaches can be developed to improve patient outcomes. The optimal dose of osimertinib for the treatment of LM disease merits further investigation since clinical benefit has been observed with both the $80 \mathrm{mg} / \mathrm{d}$ and the $160 \mathrm{mg} / \mathrm{d}$ dosing strategies. It will also be important to develop algorithms to guide when radiotherapy should be included for the treatment of CNS disease and situations where targeted therapy alone would be sufficient. In summary, the advent of potent TKIs such as osimertinib, with better CNS penetration, has resulted 
in improved outcomes for patients with oncogene-addicted NSCLC.

\section{Acknowledgments}

None.

\section{Footnote}

Conflicts of Interest: SS Ramalingam has served on scientific advisory board and received honoraria from Astra Zeneca, Amgen, Bristol Myers Squibb, Merck, Genentech/Roche, Lilly, Nektar, Loxo, Takeda and Tesaro. SS Ramalingam also receives research support (directed to the institution) from Astra Zeneca, Advaxis, Amgen, Bristol Myers Squibb, Merck, Tesaro and Takeda. JW Carlisle has no conflicts of interest to declare.

\section{References}

1. Schiller JH, Harrington D, Belani CP, et al. Comparison of four chemotherapy regimens for advanced non-smallcell lung cancer. N Engl J Med 2002;346:92-8.

2. Lee CK, Davies L, Wu YL, et al. Gefitinib or Erlotinib vs Chemotherapy for EGFR Mutation-Positive Lung Cancer: Individual Patient Data Meta-Analysis of Overall Survival. J Natl Cancer Inst 2017. doi: 10.1093/jnci/djw279.

3. Lin JJ, Cardarella S, Lydon CA, et al. Five-Year Survival in EGFR-Mutant Metastatic Lung Adenocarcinoma Treated with EGFR-TKIs. J Thorac Oncol 2016;11:556-65.

4. Ahn MJ, Tsai CM, Shepherd FA, et al. Osimertinib in patients with T790M mutation-positive, advanced nonsmall cell lung cancer:Long-term follow-up from a pooled analysis of 2 phase 2 studies. Cancer 2019;125:892-901.

5. Soria JC, Ohe Y, Vansteenkiste J, et al. Osimertinib in Untreated EGFR-Mutated Advanced Non-Small-Cell Lung Cancer. N Engl J Med 2018;378:113-25.

6. Wu YL, Ahn MJ, Garassino MC, et al. CNS Efficacy of Osimertinib in Patients With T790M-Positive Advanced Non-Small-Cell Lung Cancer:Data From a Randomized Phase III Trial (AURA3). J Clin Oncol 2018;36:2702-9.

7. Mok TS, Wu YL, Ahn MJ, et al. Osimertinib or PlatinumPemetrexed in EGFR T790M-Positive Lung Cancer. N Engl J Med 2017;376:629-40.

8. Heon S, Yeap BY, Britt GJ, et al. Development of central nervous system metastases in patients with advanced non-small cell lung cancer and somatic EGFR mutations treated with gefitinib or erlotinib. Clin Cancer Res
2010;16:5873-82.

9. Hsu F, De Caluwe A, Anderson D, et al. EGFR mutation status on brain metastases from non-small cell lung cancer. Lung Cancer 2016;96:101-7.

10. Rangachari D, Yamaguchi N, VanderLaan PA, et al. Brain metastases in patients with EGFR-mutated or ALKrearranged non-small-cell lung cancers. Lung Cancer 2015;88:108-11.

11. Magnuson WJ, Lester-Coll NH, Wu AJ, et al. Management of Brain Metastases in Tyrosine Kinase Inhibitor-Naive Epidermal Growth Factor ReceptorMutant Non-Small-Cell Lung Cancer: A Retrospective Multi-Institutional Analysis. J Clin Oncol 2017;35:1070-7.

12. Ballard P, Yates JW, Yang Z, et al. Preclinical Comparison of Osimertinib with Other EGFR-TKIs in EGFR-Mutant NSCLC Brain Metastases Models, and Early Evidence of Clinical Brain Metastases Activity. Clin Cancer Res 2016;22:5130-40.

13. Cheng H, Perez-Soler R. Leptomeningeal metastases in non-small-cell lung cancer. Lancet Oncol 2018;19:e43-55.

14. Togashi Y, Masago K, Masuda S, et al. Cerebrospinal fluid concentration of gefitinib and erlotinib in patients with non-small cell lung cancer. Cancer Chemother Pharmacol 2012;70:399-405.

15. Reungwetwattana T, Nakagawa K, Cho BC, et al. CNS Response to Osimertinib Versus Standard Epidermal Growth Factor Receptor Tyrosine Kinase Inhibitors in Patients With Untreated EGFR-Mutated Advanced NonSmall-Cell Lung Cancer. J Clin Oncol 2018. [Epub ahead of print].

16. Goss G, Tsai CM, Shepherd FA, et al. CNS response to osimertinib in patients with T790M-positive advanced NSCLC: pooled data from two phase II trials. Ann Oncol 2018;29:687-93.

17. Wu YL, Planchard D, Lu S, et al. Pan-Asian adapted Clinical Practice Guidelines for the management of patients with metastatic non-small-cell lung cancer: a CSCO-ESMO initiative endorsed by JSMO, KSMO, MOS, SSO and TOS. Ann Oncol 2019;30:171-210.

18. National Comprehensive Cancer Network. NCCN Guidelines version 1. Central nervous system cancers 2019. Available online: https://www.nccn.org/professionals/ physician_gls/pdf/cns.pdf

19. Ahn MJ, Chiu CH, Cheng Y, et al. Osimertinib for patients (pts) with leptomeningeal metastases (LM) associated with EGFRm advanced NSCLC: the AURA LM study. Ann Oncol 2019;30:ii38-68.

20. Yang JCH, Cho BC, Kim DW, et al. Osimertinib for 
patients (pts) with leptomeningeal metastases (LM) from EGFR-mutant non-small cell lung cancer (NSCLC): Updated results from the BLOOM study. J Clin Oncol 2017;35:2020.

21. ClinicalTrials.gov. 103 Studies found for: osimertinib | Lung Cancer. Accessed on April 20, 2019. Available online: https://clinicaltrials.gov/ct2/results? cond=Lung+Cancer\&t erm $=$ osimertinib \&cntry $=\&$ state $=\&$ city $=\&$ dist $=$

22. Tsao MN, Rades D, Wirth A, et al. Radiotherapeutic and surgical management for newly diagnosed brain metastasis(es):An American Society for Radiation

Cite this article as: Carlisle JW, Ramalingam SS. Improving outcomes for brain metastases in EGFR mutated NSCLC. Transl Lung Cancer Res 2019;8(Suppl 4):S355-S359. doi: 10.21037/tlcr.2019.05.08
Oncology evidence-based guideline. Pract Radiat Oncol 2012;2:210-25.

23. Badiyan SN, Regine WF, Mehta M. Stereotactic Radiosurgery for Treatment of Brain Metastases. J Oncol Pract 2016;12:703-12.

24. Camidge DR, Kim HR, Ahn MJ, et al. Brigatinib versus Crizotinib in ALK-Positive Non-Small-Cell Lung Cancer. N Engl J Med 2018;379:2027-39.

25. Peters S, Camidge DR, Shaw AT, et al. Alectinib versus Crizotinib in Untreated ALK-Positive Non-Small-Cell Lung Cancer. N Engl J Med 2017;377:829-38. 